\title{
Therapievergleich bei infiziertem Sinus pilonidalis - Unterschiede von Narbenqualität und Outcome nach Sekundärheilung oder Limberglappenplastik im Rahmen einer prospektiven Studie
}

\author{
Comparison of Treatments for an Infected Pilonidal Sinus: Differences in Scar Quality and \\ Outcome Between Secondary Wound Healing and Limberg Flap in a Prospective Study
}

Autoren

Institute

\section{S. Dahmann ${ }^{1}$, P. B. Lebo ${ }^{2}$, M. V. Meyer-Marcotty}

${ }^{1}$ KlinikumStadtSoest, Klinik für Plastische, Hand- und Wiederherstellungschirurgie, Soest ${ }^{2}$ LKH-Univ. Klinikum Graz, Klinische Abteilung für Plastische, Ästhetische und Rekonstruktive Chirurgie, Universitätsklinik für Chirurgie, Graz, Austria

\section{Schlüsselwörter \\ - Sinus pilonidalis \\ - Narbenqualität \\ - Limberglappen \\ - Sekundäre Wundheilung \\ - Hautelastizität}

Key words
pilonidal sinus
scar quality
Limberg flap
secondary wound healing
skin elasticity

eingereicht $\quad 5.9 .2015$

akzeptiert $\quad 30.11 .2015$

Bibliografie

DOI http://dx.doi.org/

10.1055/s-0041-111322

Handchir Mikrochir Plast Chir

2016; 48: 111-119

(c) Georg Thieme Verlag KG Stuttgart $\cdot$ New York

ISSN 0722-1819

Korrespondenzadresse

\section{Dr. Sonja Dahmann}

Klinikum Stadt Soest Plastische Chirurgie

Senator-Schwartz-Ring 8

59494 Soest

dahmann@klinikumstadtsoest.de

\section{Zusammenfassung}

\section{$\nabla$}

Hintergrund: Nach der Exzision eines infizierten Sinus pilonidalis gibt es verschiedene Therapieoptionen des Defekts. Wir verglichen Sekundarheilung und Limberglappenplastik hinsichtlich Narbenqualität und Beschwerden, Rezidivhäufigkeit, Arbeitsunfähigkeit und Patientenzufriedenheit 1 Jahr postoperativ.

Methodik: Von 55 Patienten, welche 20112012 im KlinikumStadtSoest an einem Sinus pilonidalis operiert wurden, konnten 16 Patienten mit sekundärer Wundheilung und 17 Patienten mit Limberglappenplastik eingeschlossen werden. Insbesondere interessierten uns die Objektivierbarkeit der Narbenqualität und -elastizität durch die Parameter der Hautdehnbarkeit und -verschieblichkeit. Hierzu kam eine von uns entwickelte Messmethode zur Ermittlung des Sakral-Lumbalen Hautdehnungsquotienten (SL-Quotient) und der Sakralen Hautverschieblichkeit zum Einsatz. 100 gesunde Probanden dienten als Kontrollgruppe. Die Schmerzskalen, Rezidivhäufigkeit, Zufriedenheit der Patienten mit Verlauf und kosmetischem Ergebnis sowie die Dauer der Arbeitsunfähigkeit wurden ebenfalls ermittelt.

Ergebnisse: Der Sakral-Lumbale Hautdehnungsquotient fiel in der Limberggruppe signifikant besser aus, als in der Sekundärheilungsgruppe. Bezüglich der Verschieblichkeit gab es eine deutliche Tendenz zu günstigeren Werten in der Limberggruppe. Die Dehnbarkeit und Verschieblichkeit in der Limberggruppe entsprach den Referenzwerten aus der gesunden Probandengruppe. Die Dehnbarkeit nach sekundärer Wundheilung war hingegen signifikant $(p=0,001)$ schlechter als in der gesunden Referenzgruppe. Die Dauer der Arbeitsunfähigkeit nach der Lappenplastik war in der Limberggruppe (MW 29 Tage) signifikant $(\mathrm{p}=0,02)$ geringer als die Dauer nach der Sinusexzision in der Gruppe mit sekundärer Wundheilung (MW 63 Tage). Bezüglich

\section{Abstract \\ $\nabla$}

Objective: There are various options for wound treatment after the excision of a pilonidal sinus. The aim of our study was to compare secondary healing to Limberg flap wound closure, with a focus on scar quality and patient complaints, rate of recurrence, period of absence from work as well as functional and aesthetic results one year after surgery.

Method: 33 out of 55 patients who underwent pilonidal sinus excision in our department (KlinikumStadtSoest, Soest, Germany) between 2011 and 2012 were enrolled in the study. 16 of these 33 patients had chosen secondary wound healing and 17 were treated with a Limberg flap for defect coverage. First and foremost, we aimed to objectify scar quality and elasticity by measuring the parameters of skin distensibility and mobility. To this end, we used a self-developed method to ascertain the sacral lumbar skin distension quotient (SL quotient) as well as sacral skin mobility. 100 healthy volunteers served as a control group. Also we collected information about pain, time of absence from work and frequency of recurrence and asked patients about their satisfaction with the functional and aesthetic results.

Results: The results for the sacral lumbar skin distension quotient were significantly better after Limberg flap wound closure compared with secondary wound healing. As regards distensibility, there was a marked trend to more favourable values in the Limberg group. No differences in distensibility and mobility were observed between the Limberg group and the control group, whereas skin distensibility was significantly reduced $(p=0.001)$ in secondary healing compared with the control group. Time off work was significantly longer in secondary healing (mean 63 days) than after Limberg flap (mean 29 days). No differences were identified regarding patient satisfaction, pain scores and frequency of recurrence. 
der Schmerzen, Zufriedenheit mit Verlauf und kosmetischem Ergebnis sowie Anzahl der Rezidive zeigten sich keine Unterschiede zwischen den Patientengruppen.

Schlussfolgerung: Durch den Wundverschluss mittels Limberglappenplastik nach Exzision eines infizierten Sinus pilonidalis kann nicht nur die Dauer der Arbeitsunfähigkeit reduziert werden, sondern auch eine Narbe entstehen, die besser verschieblich und dehnbar ist, im Vergleich zur Sekundärheilung. Schmerzen und Zufriedenheit waren in beiden Gruppen sehr günstig, ohne Gruppenunterschiede. Folgestudien an einem größeren Patientenkollektiv und mit längerem Follow-up sind geplant. Wir stellen unseren Patienten zunächst weiterhin beide Therapieoptionen als mögliche Alternativen dar.

\section{Einleitung}

$\nabla$

Thema

Für die Behandlung eines akut abszedierten Sinus pilonidalis gibt es die eindeutige Empfehlung der Exzision durch eine S3-Leitlinie [1]. Doch für die weitere Therapie des immer resultierenden Hautweichteildefektes ist bislang noch kein Goldstandard etabliert. Die verschiedenen Möglichkeiten von konservativen und operativen Therapiemethoden werden im Nachfolgenden dargestellt. Es stellt sich hierbei die Frage, welche Folgen in Abhängigkeit der Therapiewahl für den Patienten entstehen.

Ziel der vorliegenden Studie ist der Vergleich von konservativer und operativer Therapie hinsichtlich subjektiver Bewertung von Schmerzen und Zufriedenheit mit dem Verlauf und ästhetischem Ergebnis und andererseits auch objektivierbarer Daten zur Narbenqualität, Dauer der Arbeitsunfähigkeit und Rezidivhäufigkeit bei diesen Differentialtherapien zu erheben.

\section{Sinus pilonidalis}

Der Sinus pilonidalis ist eine entzündliche Erkrankung der Sakralregion, welche asymptomatisch, akut abszedierend oder chronisch verlaufen kann. Die Ätiologie ist noch nicht abschließend geklärt. Diskutiert wird ein multifaktorielles Geschehen bei welchem in die Tiefe eindringende Haare [2], mangelnde Hygiene [3] und auch eine genetische Disposition eine Rolle spielen $[1,2,4]$. Als weitere Risikofaktoren wurden Adipositas, Hormone, lokale Verletzungen und Reibung identifiziert [5,6].

Der Altersgipfel liegt im jungen Erwachsenenalter [7]. Diese Tatsache ist ein entscheidender Faktor vor dem Hintergrund einer ggf. langwierigen Behandlung mit entsprechend langer Arbeitsunfähigkeit bei diesen Patienten. 48/100000 Einwohner sind in Deutschland betroffen, wobei die Erkrankung bei Männern 2,2 mal häufiger auftritt als bei Frauen [8]. In anderen Ländern tritt der Pilonidalsinus jedoch wesentlich häufiger auf, so sind in der Türkei einschließlich asymptomatischer Fälle 8,3\% der Bevölkerung betroffen [9].

Anhand der klinischen Untersuchung wird die Diagnose gestellt. Es findet sich bei der asymptomatischen Form ein reizloser Porus oder mehrere davon in der Rima ani, welche bei der chronischen Form meist blutig-serös sezernieren. Schmerzen und Schwellung treten bei akuter Abszedierung auf. Auf eine Bildgebung kann in der Regel verzichtet warden [1]. Mit einer Spontanheilung ist nicht zu rechnen, ein Übergang von der asymptomatischen in die akut abszedierte oder die chronische Form ist möglich [1].

Zur Therapie des Sinus pilonidalis gibt es aktuelle Empfehlungen in Form einer S3-Leitlinie. Für die asymptomatische Form ist keine Behandlung notwendig. Bei einer akuten Abszedierung
Conclusion: Wound closure via Limberg flap after the excision of an infected pilonidal sinus not only helps to reduce absence from work, but also produces a scar which is more distensible and movable compared with secondary healing. Patient satisfaction and pain scores were very good in both groups, with no differences observed by us. We are planning to collect more data with a bigger sample of patients and a longer follow-up period in future studies. For the time being, we will continue to provide both treatment methods to our patients. herrscht Konsens über die Notwendigkeit der chirurgischen Entlastung, wenn möglich durch eine definitive Exzision [1]. Für das weitere Verfahren mit dem entstandenen Hautweichteildefekt oder für den chronischen Pilonidalsinus gibt es keine einheitliche Empfehlung $[1,10,11]$. Unterschieden wird zwischen minimalinvasiven Verfahren (Phenol-Instillation [12], Pit Pricking [13], Sinusektomie [14]), offener Wundbehandlung [15,16], Primärnaht [11] und plastisch chirurgischen paramedianen Verfahren (v.a. Limberglappenplastik [17], Karydakislappenplastik $[18,19]$, Cleft-lift-Verfahren [20], Z-Plastik [21,22], bilateraler adipofascialer Transpositionallappen [23], V-Y-Lappenplastik [24]). Metaanalysen zufolge ist die Sekundärheilung lediglich der Primärnaht hinsichtlich der Rezidivrate überlegen [10]. Aufgrund der hohen Rezidiv- und Komplikationsrate sollte von einer Primärnaht eher Abstand genommen warden [10]. Off-Midline Verfahren sind Aufgrund geringerer Rezidiv- und Komplikationsraten sowie kürzerer Heilungszeiten günstiger als Mittelliniennähte $[10,11]$.

Um das Langzeitergebnis zu beurteilen wurde vorwiegend die Rezidivquote $[25,26]$ und in einer Einzelstudie [8] auch die Zufriedenheit mit dem optischen Ergebnis herangezogen. In unserer vorliegenden Studie wird das Augenmerk nun zusätzlich auf die Narbenqualität gelegt.

\section{Narben}

Narben am Rücken haben die Tendenz breiter zu werden, je kaudaler sie lokalisiert sind [27]. Dadurch kommt der Schnittführung und Ausdehnung der Narbe im Sakralbereich eine besondere Bedeutung zu. Berry et al. zeigten in ihrer in vivo Studie an sekundär heilenden menschlichen Pilonidalsinuswunden, dass der Anteil an Narbengewebe nur 12 Prozent des ursprünglichen Wundvolumens ausmacht [28]. Anders als z.B. in Extremitätenwunden trägt in der Sakralregion hauptsächlich eine durch speziell differenzierte Myofibroblasten vermittelte Kollagenkontraktion zum Wundverschluss bei. Hierdurch werden $88 \%$ des Defektvolumens durch das umgebende Dermis- und Fettgewebe aufgefüllt, welches in die Wunde hineingezogen wird [28]. Die Narbe steht demnach unter Spannung. Das in der Mittellinie befindliche Narbengewebe ist durch einen sinkenden Gehalt oder die Desorganisation elastischer Fasern gekennzeichnet $[29,30]$ sowie durch eine Verdichtung der Kollagenfibrillen zu dicken Kollagenfasern [28], vorwiegend vom Kollagen-Typ I [31].

Auch beim plastisch chirurgischen Wundverschluss kommt es zu einer Vernarbung, jedoch muss hier kein Volumendefekt mit Narbengewebe aufgefüllt werden, wie bei der Sekundärheilung. Das Volumendefizit wird bei Lappenplastiken durch gesundes dermoadipofasciales Gewebe ausgeglichen. 
Zum Vergleich der Narben nach sekundärer Wundheilung mit Narben nach Limberglappenplastik beurteilten wir daher die Elastizität der Haut in der Region des ehemaligen Pilonidalsinus. Wir entwickelten hierzu eine Messmethode der Hautdehnbarkeit und -verschieblichkeit (s.u.). im Rahmen einer eigenen Studie, welche bereits veröffentlicht wurde [32].

\section{Methodik}

\section{$\nabla$}

Bei 55 Patienten wurde im Zeitraum von 2011-2012 im KlinikumStadtSoest ein akut abszedierter Sinus pilonidalis in der Abteilung für Viszeralchirurgie exzidiert. Allen Patienten wurde nach ausführlicher Aufklärung die Therapiemöglichkeiten durch offene Wundbehandlung oder durch Rekonstruktion mittels Limberglappenplastik in unserer Abteilung für Plastische Chirurgie angeboten. Die Wahl des nachfolgend dargestellten Verfahrens wurde jedem Patienten überlassen.

\section{Offene Wundbehandlung, Gruppe 1}

Der sakrale Weichteildefekt wurde täglich mit Octenisept ${ }^{\circledR}$ (Wirkstoffe Octenidindihydrochlorid und Phenoxyethanol, Firma Schülke) desinfiziert, mit NaCl-Lösung ausgespült und anschließend mit Jodoformgaze tamponiert ( $\bullet$ Abb. 1a, b), gefolgt von einer Abdeckung mit sterilen Kompressen und Klebefixierung (Fixomull ${ }^{\circledR}$, Firma BSN medical). Diese Behandlung erfolgte zu Beginn in der Ambulanz unseres Krankenhauses und wurde später vom Hausarzt fortgeführt.

\section{Limberglappenplastik, Gruppe 2}

In einem zweiten Klinikaufenthalt innerhalb von 10 Tagen nach der Abszesssanierung erfolgte die Operation in Bauchlage und Allgemeinanästhesie nach präoperativer Darmreinigung mittels Abführmaßnahmen. Die Wundränder wurden so exzidiert, dass ein vertikal ausgerichteter, rhombusförmiger Defekt entstand, optimalerweise mit gleicher Seitenlänge und dem gleichen Winkel der gegenüberliegenden Ecken von 60 und $120^{\circ}(\bullet$ Abb. 2a). Nun wurde die Lappenschnittführung geplant ( $\bullet$ Abb. 2b, $\bullet$ 3a), indem die horizontale Winkelhalbierende mit einer eingezeichneten Linie um die Seitenlänge A verlängert wurde. Am Ende dieser Linie wurde im Winkel $\alpha$ eine Linie der Seitenlänge B ein-

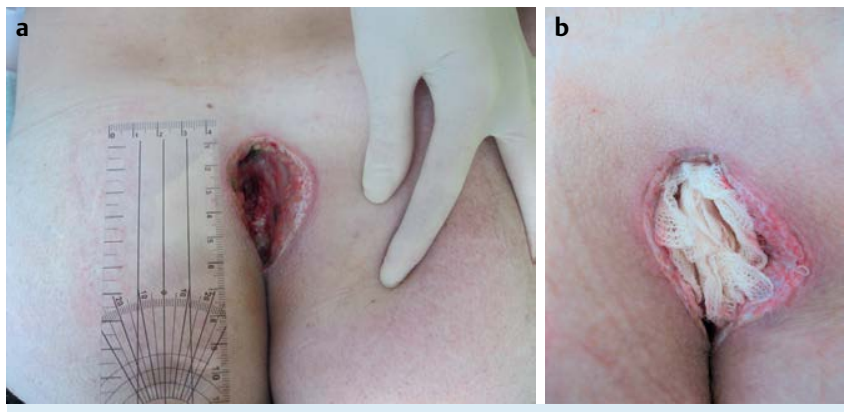

Abb. 1 a: Weichteildefekt nach Sinus pilonidalis Exzision b Wundtamponage gezeichnet ( $\bullet$ Abb. 2a). Entlang der Linien erflogte nun die Lappenhebung einschließlich Dermis, Fettgewebe und Glutealfaszie ( $\bullet$ Abb. 3b). Der Lappen wurde nach Mobilisation der Wundränder in den Defekt hineingeschwenkt ( $\bullet$ Abb. 3c) und nach Redondrainageneinlage durch Faszien-, Subkutan- und Hautnähte fixiert ( $\odot$ Abb. 3d). Anschließend erfolgte das Aufbringen von medizinischem Sprühkleber, ein Druckverband sowie die Anlage eines Kompressionsgurtes. Die Drainage wurde i.d.R. am 2. postoperativen Tag entfernt.

\section{Datenerhebung}

Alle 55 Patienten, die in den Jahren 2011 und 2012 im KlinikumStadtSoest an einem Sinus pilonidalis operiert wurden, kontaktierten wir im Rahmen dieser Studie. Von diesen Patienten wurden 33 im Zeitraum von Mai 2012 bis August 2013 zur Anamnese und Untersuchung einbestellt und mit ihrem schriftlichen Einverständnis in die Studie eingeschlossen.

Es wurden epidemiologische Daten erhoben, wie Alter, Geschlecht, Gewicht und Größe. Das Rauchverhalten wurde erfasst, indem eine Zuordnung zu den Gruppen Raucher, ehemaliger Raucher, Nichtraucher erfolgte und die Anzahl der täglich konsumierten Zigaretten erfragt wurde.

Die Zufriedenheit mit dem Behandlungsverlauf und dem Aussehen der Narbe wurde auf einer Skala von „0-5“ von den Patienten angegeben, wobei „0“ die schlechteste und „5“ die bestmögliche Bewertung war. Aktuelle Schmerzen in Ruhe und bei Belastung wurden anhand der numerischen Analogskala von „0“ bis „10“ erfasst. Die Dauer der Arbeitsunfähigkeit insgesamt und die Zeit bis zur Arbeitswiederaufnahme nach der letzten Operation wurden dokumentiert, ebenso wie seither aufgetretene Rezidive.

Im Rahmen dieser Studie interessierten uns insbesondere die Objektivierbarkeit der Narbenqualität und -elastizität durch die Parameter der Hautdehnbarkeit und -verschieblichkeit. Hierzu kam eine von uns entwickelte, an 100 gesunden Probanden evaluierte Messmethode zur Ermittlung des Sakral-Lumbalen Hautdehnungsquotienten (SL-Quotient) und der Sakralen Hautverschieblichkeit zum Einsatz [32]. Zusätzlich erfolgte eine Fotodokumentation der Narbe und eine Bewertung von Farbe (hyperpigmentiert, umgebungsneutral, hypopigmentiert), Relief (erhaben, eben oder eingesunken) und Konsistenz (weich, mittelfest, derb).
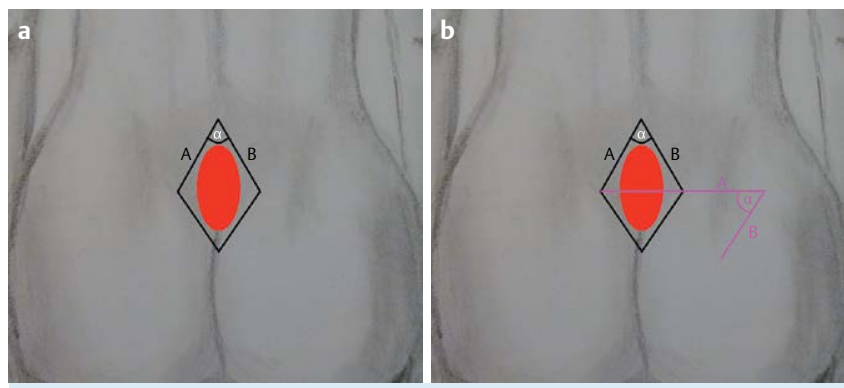

Abb. 2 a Rhombusförmige Exzision (Skizze) b Planung der Limberglappenplastik (Skizze)
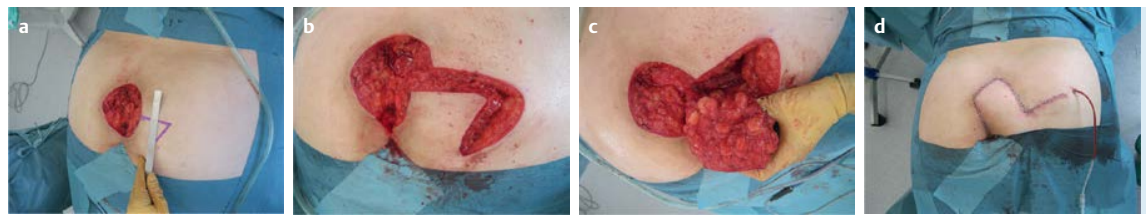

Abb. 3 a: Lappenplanung b: Lappenumschneidung. c: Lappenschwenkung d: Einnaht in den Defekt 


\section{Messung der Dehnbarkeit}

Zur Messung der Hautdehnbarkeit stand der Patient aufrecht mit dem Rücken zum Untersucher. Auf dem Rücken des Patienten wurden 4 Punkte (A, B, C und D) vertikal übereinander markiert. Der unterste Punkt A befand sich dabei am Beginn der Rima ani. $10 \mathrm{~mm}$ kranial lag der Punkt B, $100 \mathrm{~mm}$ oberhalb von Punkt B wurde der Punkt C angezeichnet. Der höchste Punkt D lag erneut $10 \mathrm{~mm}$ kranial von Punkt C ( $\odot$ Abb. 4a). Nun wurde der Patient gebeten sich soweit mit dem Oberkörper nach vorne zu beugen, bis er bei ausgestreckten Armen und Beinen auf beiden Seiten mit dem Handballen die Mitte seiner Patella erreichte. Die Strecken $A B$ und $C D$ wurden nun erneut gemessen und notiert ( 0 Abb. 4b).

Zusätzlich wurde der Quotient zwischen den gedehnten Strecken $A B$ und $C D$ gebildet („AB/CD“). Hierdurch erhielt man eine Relation zwischen der Dehnbarkeit der sakralen und der lumbalen Haut im selben Individuum. Die Strecke AB befand sich im Bereich des ehemaligen Sinus pilonidalis, wobei die Strecke CD sich im Lumbalbereich außerhalb der Narbenregion befand und als gesunde Referenz diente [32].

\section{Messung der Verschieblichkeit}

Für die Messung der Hautverschieblichkeit stand der Patient aufrecht. Mit dem rechten Zeigefinger des Untersuchers schob dieser die Haut bei Punkt A soweit wie möglich nach kranial $(\bullet$ Abb. 5a). Der Ort an dem sich nun Punkt $A$ befand, wurde als A' bezeichnet. Sodann wurde die Distanz von A nach A' gemes$\operatorname{sen}(\mathbf{O}$ Abb. 5b).

\section{Auswertung}

Die statistische Auswertung erfolgte mit dem Programm SPSS ${ }^{\circledR}$ (Statistical Package for the Social Sciences, IBM Corp. Ausgabe 2012. IBM SPSS Statistics for Windows, Version 21.0. Armonk,
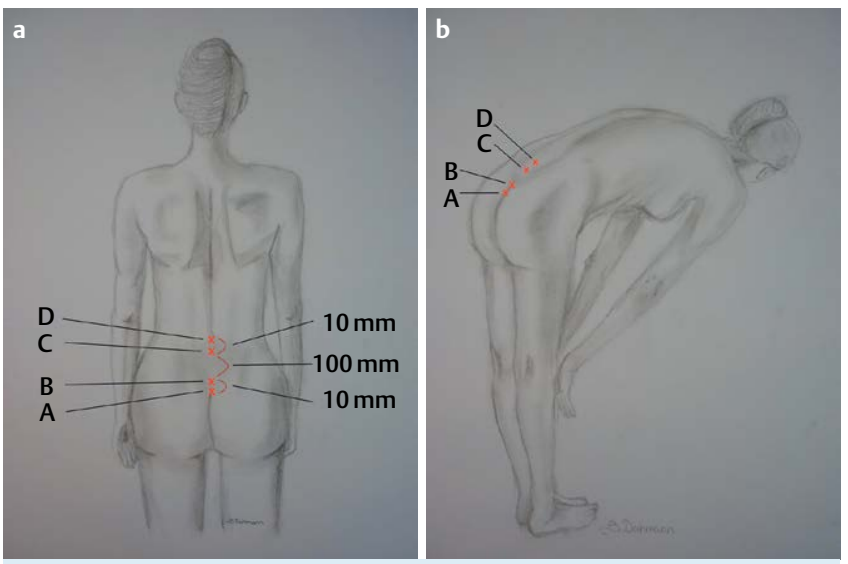

Abb. 4 a: Positionierung der Markierungspunkte im Stehen b Körperhaltung zur Messung der Hautdehnung
NY: IBM Corp.). Es wurde eine deskriptive Statistik durchgeführt (Mittelwerte, Mediane, Standardabweichungen, Quartilen), wie auch Kolmogorov-Smirnov Tests zur Berechnung der Datenverteilung, T- und Mann-Whitney-U-Tests sowie Korrelationsanalysen (Spearman).

\section{Ergebnisse}

$\nabla$

Von den 55 Patienten, die im Zeitraum von 2011 und 2012 am KlinikumStadtSoest eine Sinus pilonidalis Exzision erhalten hatten, wurde ein Patient ausgeschlossen, da bei ihm in einem auswärtigen Krankenhaus eine plastisch chirurgische Defektdeckung durchgeführt wurde. Ein weiterer Patient wurde ausgeschlossen, da er aufgrund vieler Rezidive zuvor bereits verschiedene Lappenplastiken in der Sakralregion erhalten hatte. Weitere 20 Patienten konnten nicht erreicht oder nicht zur Studienteilnahme motiviert werden. Es wurden 16 Patienten mit sekundärer Wundheilung (Gruppe 1) und 17 Patienten mit Limberglappenplastik (Gruppe 2) in die Studie eingeschlossen. In Gruppe 1 betrug der Männeranteil 72,2\%, in Gruppe 2 waren es $81,8 \%$. Der Altersdurchschnitt in Gruppe 1 lag bei 30 Jahren und in Gruppe 2 bei 28 Jahren. Die Gruppe 1 wies einen mittleren BMI von 28,35 kg/m $\mathrm{m}^{2}$ auf. Der mittlere BMI in der Gruppe 2 lag bei $27 \mathrm{~kg} / \mathrm{m}^{2}$. In Gruppe 1 trat in $8 \%$ ein Rezidiv auf, die Rezidivrate in Gruppe 2 lag bei $9 \%$. Hier bestand kein signifikanter Unterschied. Zum Vergleich der Hautelastizität wurden 105 gesunde Probanden Im KlinikumStadtSoest zur freiwilligen Studienteilnahme rekrutiert. Drei davon wurden ausgeschlossen, da sie zuvor Operationen im Sakralbereich hatten. Ein Proband wurde aufgrund langstreckiger Narben am Rücken und eine Probandin aufgrund mangelnder Wirbelsäulenbeweglichkeit bei Lumbalgie ausgeschlossen. Unter den 100 eingeschlossenen Studienteilnehmern (Gruppe 3) waren 43 Männer und 57 Frauen im Alter von 16 bis 82 Jahren. Der BMI variierte zwischen 17 und $46 \mathrm{~kg} / \mathrm{m}^{2}$ mit einem Durchschnitt von $25 \mathrm{~kg} / \mathrm{m}^{2}$.

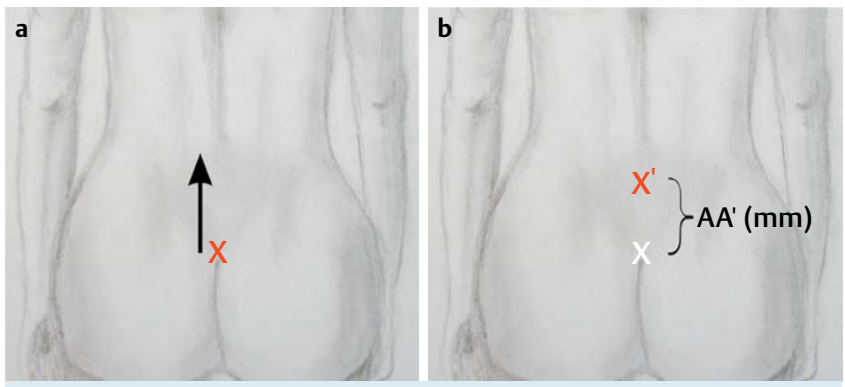

Abb. 5 a: Markierung am kranialen Ende der Rima ani und Schubrichtung b Messung der Verschieblichkeit

Tab. 1 deskriptive Statistik der epidemiologischen Voraussetzungen.

\begin{tabular}{|c|c|c|c|c|c|c|c|c|c|c|c|c|}
\hline & \multicolumn{3}{|c|}{ Sekundärheilung } & \multicolumn{3}{|c|}{ Limberglappen } & \multicolumn{3}{|c|}{ Gesund } & \multicolumn{3}{|c|}{ p-Wert } \\
\hline & MW & Med & SD & MW & Med & SD & MW & Med & SD & $\begin{array}{l}\text { Sekudär-heilung } \\
\text { vs. Limberg. }\end{array}$ & $\begin{array}{l}\text { Sekundär-heilung vs. } \\
\text { Gesund }\end{array}$ & $\begin{array}{l}\text { Limberg vs. } \\
\text { Gesund }\end{array}$ \\
\hline Alter & 30 & 23 & 13 & 28 & 26 & 13 & 36 & 32 & 14 & 0,558 & 0,068 & $0,014^{*}$ \\
\hline Größe & 177 & 176 & 11 & 178 & 180 & 9 & 173 & 173 & 11 & 0,854 & 0,140 & $0,041^{*}$ \\
\hline Gewicht & 90 & 89 & 17 & 86 & 85 & 14 & 76 & 73 & 19 & 0,547 & $0,003^{*}$ & $0,003^{*}$ \\
\hline $\mathrm{BMI}$ & 28,35 & 27,45 & 4,38 & 27,25 & 27,10 & 4,41 & 25,17 & 24,30 & 5,18 & 0,591 & $0,010^{*}$ & $0,022^{*}$ \\
\hline
\end{tabular}

MW $=$ Mittelwert, Med $=$ Median, SD $=$ Standardabweichung, ${ }^{*}=$ statistisch signifikant 


\section{Epidemiologie}

Die Patientengruppen 1 und 2 unterschieden sich nicht in Alter, BMI, Größe (Gruppe1: MW $177 \mathrm{~cm}$; Gruppe 2: MW $178 \mathrm{~cm}$ ) oder Gewicht (Gruppe1: MW $90 \mathrm{~kg}$; Gruppe 2: MW $86 \mathrm{~kg}$ ) (๑ Tab. 1). Gruppe 1 und 2 wiesen höhere Werte von BMI (Gruppe1 vs. Kontrollgruppe $p=0,01$; Gruppe 2 vs. Kontrollgruppe: $p=0,022$ ) und Gewicht auf (Gruppe 1 vs. Kontrollgruppe $\mathrm{p}=0,003$; Gruppe 2 vs. Kontrollgruppe 2: $p=0,003$ ). Zwischen der Limberggruppe und der Kontrollgruppe bestanden zusätzlich signifikante Differenzen in Alter $(p=0,014)$ und Größe $(p=0,041)$.

\section{Rauchverhalten}

In der Sekundärheilungsgruppe waren 69,2\% Raucher, diese rauchten im Durchschnitt 11,78 Zigaretten pro Tag. In der Gruppe 2 waren 50\% Raucher, diese rauchten durchschnittlich 12,36 Zigaretten pro Tag ( $\bullet$ Tab. 2). Im Chi-Quadrat Test wurde ein Zusammenhang zwischen dem Rauchverhalten (ja/nein) und der Gruppe ausgeschlossen $(p=0,458)$. Im U-Test zeigte sich, dass kein Unterschied bezüglich der Anzahl von täglich konsumierten Zigaretten zwischen den Gruppen bestand $(\mathrm{p}=0,816)$.

\section{Schmerzen}

Für beide Patientengruppen ergab sich ein mittlerer Ruheschmerz von 0 und ein Belastungsschmerz von 2 auf der numerischen Analogskala. Der Mann-Whitney U-Test wies demnach keinen Unterschied zwischen den Patientengruppen auf (Ruheschmerz $\mathrm{p}=0,981$, Belastungsschmerz $\mathrm{p}=0,722$ ).

Bis zum ersten schmerzfreien Gehen vergingen in der Gruppe 1 durchschnittlich 79 und in der Gruppe 213 Tage. Bei einer sehr hohen Standardabweichung war dieser Unterschied nicht signi-

Tab. 2 Rauchverhalten.

\begin{tabular}{|c|c|c|c|}
\hline Gruppe & \% Raucher & $\begin{array}{l}\text { Zig./Tag gesamt } \\
\text { MW (SD) }\end{array}$ & $\begin{array}{l}\text { Zig./Tag nur der } \\
\text { Raucher MW (SD) }\end{array}$ \\
\hline Sekundärheilung & $69,2 \%$ & $8,2(8,1)$ & $11,78(7,12)$ \\
\hline $\begin{array}{l}\text { Limberglappen- } \\
\text { plastik }\end{array}$ & $50 \%$ & $6,2(7,9)$ & $12,36(6,83)$ \\
\hline p-Wert & 0,458 & 0,484 & 0,854 \\
\hline
\end{tabular}

$\mathrm{MW}=$ Mittelwert, SD = Standardabweichung, $Z$ ig. $=$ Zigaretten fikant ( $p=0,241)$. Der schmerzfreie Toilettengang war in der Gruppe 1 nach 30 Tagen und der Gruppe 2 nach 4 Tagen möglich. Dieser Unterschied war nicht signifikant $(p=0,441)$. Die Mittelwerte sind in $\odot$ Tab. 3 dargestellt.

\section{Arbeitsausfall}

Betrachtet man die Arbeitsunfähigkeitsdauer nach der Defektdeckung, so finden sich deutlich geringere Zeiten in der Limberglappengruppe (MW 29 Tage) als in der Sekundärheilungsgruppe (MW 63 Tage). Dieser Unterschied erwies sich im Mann-Whitney U-Test als signifikant $(\mathrm{p}=0,02)$.

\section{Zufriedenheit}

Die Patientenzufriedenheit mit dem Verlauf war in Gruppe 1 (MW 3,88) und Gruppe 2 (MW 4,23) hoch, ohne einen signifikanten Gruppenunterschied im Mann-Whitney U-Test $(p=0,42)$. Für die Zufriedenheit mit dem kosmetischen Ergebnis ergaben sich für die Sekundärheilungsgruppe durchschnittlich 4,00 von 5 maximalen Punkten und für die Limberggruppe 3,59 von 5 Punkten. Es ergab sich auch hierfür kein signifikanter Unterschied $(\mathrm{p}=0,295)$.

\section{Narbenqualität}

\section{Absolute Dehnung $A B$ und $C D$}

Die Dehnung der Strecke AB, welche sich in der Sinus pilonidalis Region befindet, wies zwischen allen 3 Gruppen signifikante Unterschiede auf (Gruppe 1 vs. Gruppe 2: $p=0,035$; Gruppe 1 vs. Gruppe $3 p=0,000$; Gruppe 2 vs. Gruppe $3 p=0,038$ ), mit den niedrigsten Werten in der Gruppe $1(\mathrm{MW} 11 \mathrm{~cm})$, darauffolgend der Gruppe $2(\mathrm{MW} 12 \mathrm{~cm}$ ) und den höchsten Werten in der gesunden Kontrollgruppe (MW $13 \mathrm{~cm}$ ).

Die gesunde Referenzhaut im Bereich der Strecke CD dehnte sich in allen Gruppen ähnlich ( $\bullet$ Tab. 4).

\section{Sakral-lumbaler Hautdehnungsquotient AB/CD (SLQ)} Im Mann-Whitney U-Test zeigte sich, dass sich Limberg- und sekundäre Wundheilungsgruppe signifikant bezüglich der relativen Dehnbarkeit (SLQ) unterschieden ( $p=0,004)$, wobei die Limberglappengruppe eine höhere Dehnungsfähigkeit (MW 86\%) als die Gruppe mit Sekundärheilung (MW 75\%) aufwies.

Tab. 3 Schmerzen.

\begin{tabular}{lccccc} 
& \multicolumn{2}{c}{ Sekundärheilung } & \multicolumn{2}{c}{ Limberglappen } & p-Wert \\
& Mittelwert & Standardabweichung & Mittelwert & Standardabweichung \\
Ruheschmerz (NAS) & 0 & 1 & 0 & 0 & 0,434 \\
Belastungsschmerz (NAS) & 2 & 3 & 2 & 1 & 0,675 \\
Schmerzfreies Gehen (Tage) & 79 & 117 & 13 & 10 & 0,121 \\
Schmerzfreier Toilettengang (Tage) & 30 & 48 & 4 & 5 & 0,131
\end{tabular}

Tab. 4 Hautelastizität.

\begin{tabular}{|c|c|c|c|c|c|c|c|c|c|}
\hline & \multicolumn{6}{|c|}{ Gruppe } & \multicolumn{3}{|c|}{ p-Wert } \\
\hline & \multicolumn{2}{|c|}{ Sekundärheilung } & \multicolumn{2}{|c|}{ Limberglappen } & \multicolumn{2}{|c|}{ Gesund } & \multirow{2}{*}{$\begin{array}{l}\text { Sekundär-heilung vs. } \\
\text { Limberglappen }\end{array}$} & \multirow{2}{*}{$\begin{array}{l}\text { Sekundär-heilung } \\
\text { vs. Gesund }\end{array}$} & \multirow{2}{*}{$\begin{array}{l}\text { Limberglappen } \\
\text { vs. Gesund }\end{array}$} \\
\hline & $\mathrm{MW}(\mathrm{cm})$ & SD & $\mathrm{MW}(\mathrm{cm})$ & SD & $\mathrm{MW}(\mathrm{cm})$ & SD & & & \\
\hline$A B$ & 11 & 1 & 12 & 2 & 13 & 1 & $0,035^{*}$ & $0,000 *$ & $0,038^{*}$ \\
\hline$C D$ & 15 & 2 & 14 & 2 & 15 & 2 & 0,124 & 0,789 & 0,079 \\
\hline SL-Quotient & 0,75 & 0,11 & 0,86 & 0,08 & 0,87 & 0,11 & $0,006^{*}$ & $0,001^{*}$ & 0,683 \\
\hline Verschieblichkeit & 14 & 8 & 16 & 8 & 16 & 6 & 0,165 & $0,018^{*}$ & 0,279 \\
\hline
\end{tabular}

$\mathrm{MW}=$ Mittelwert, $\mathrm{SD}=$ Standardabweichung, ${ }^{*}=$ statistisch signifikant 
Hierbei entsprach die Dehnungsfähigkeit der Limberggruppe der gesunden Referenzgruppe (MW 87\%, U-Test: $\mathrm{p}=0,68$ ), wobei die Sekundärheilungsgruppe im Vergleich zur Referenzgruppe einen signifikant niedrigeren SLQ aufwies $(p=0,001)$ ( $\bullet$ Abb. 6). Der SLQ wurde durch Alter, BMI, Größe oder Gewicht nicht beeinflusst.

\section{Verschieblichkeit AA'}

Im direkten Vergleich der beiden Patientengruppen zeigte sich kein signifikanter Unterschied der Hautverschieblichkeit (SH: MW $14 \mathrm{~cm}$, LP: MW $16 \mathrm{~cm}$; p=0,165) Von der gesunden Kontrollgruppe (MW $16 \mathrm{~cm}$ ) unterschied sich nur die Sekundärheilungsgruppe signifikant $(p=0,018)(\bullet$ Abb. 7).

Als Biasfaktor erwiesen sich der BMI (Korrelationskoeffizient $r=0,30 ; p=0,001$ ) und das Gewicht (Korrelationskoeffizient $r=0,27 ; p=0,002)$ wie aus der vorstehenden Tabelle ersichtlich.

\section{Narbenbeschaffenheit}

Die Narbe war in der Limberglappengruppe vorwiegend hyperpigmentiert (58\%), während in der Sekundärheilungsgruppe

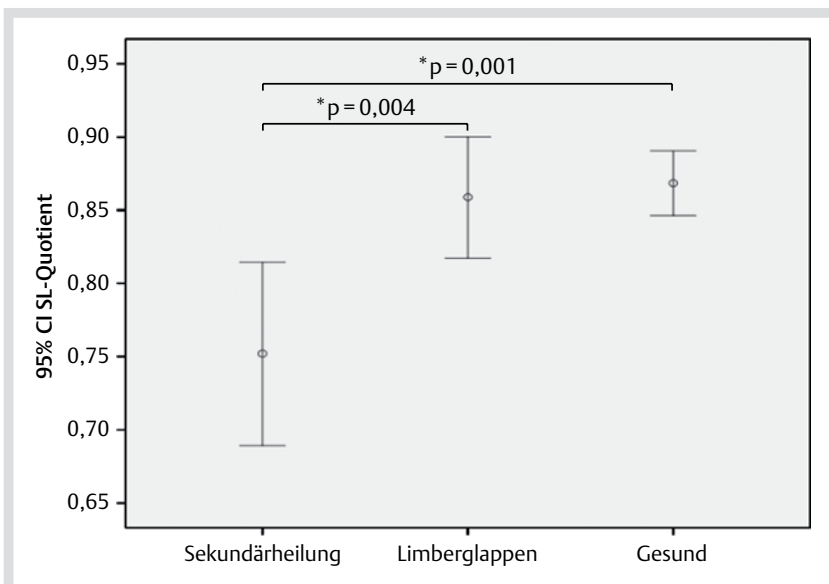

Abb. 6 SLQ

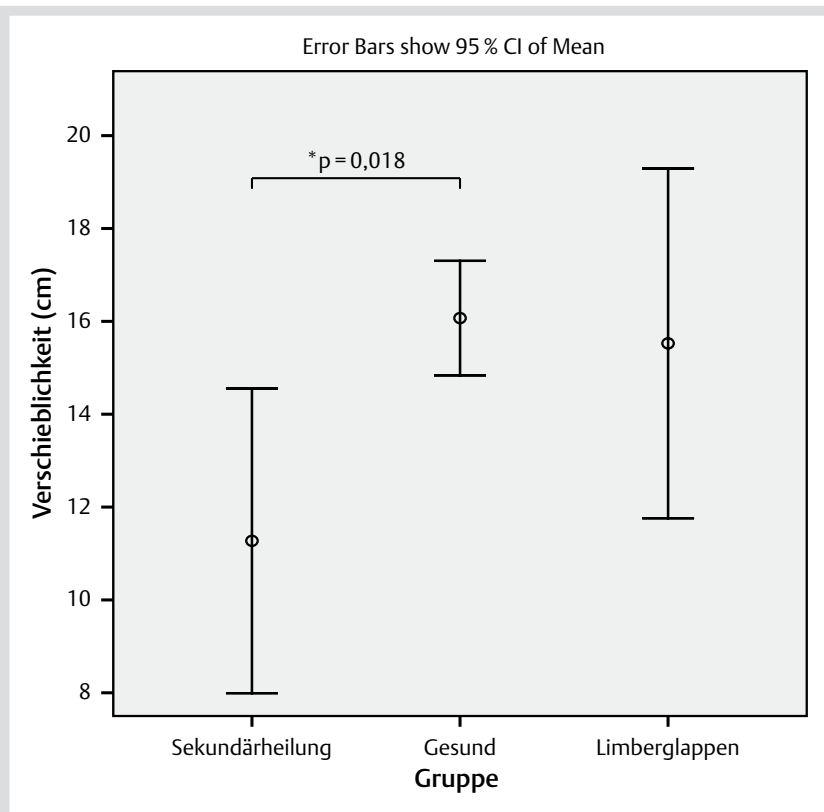

Abb. 7 Verschieblichkeit
Tab. 5 Narbenbeschaffenheit.

\begin{tabular}{|c|c|c|c|}
\hline & & \multicolumn{2}{|c|}{ Prozent \% } \\
\hline & & Sekundärheilung & Limberglappen \\
\hline \multirow[t]{3}{*}{ Farbe } & hyperpigmentiert & 33,3 & 57,9 \\
\hline & Hypopigmentiert & 26,7 & 5,3 \\
\hline & Idem & 40,0 & 36,8 \\
\hline \multirow[t]{3}{*}{ Relief } & Eingesunken & 53,3 & 15,8 \\
\hline & Erhaben & 6,7 & 36,8 \\
\hline & Idem & 40,0 & 47,4 \\
\hline \multirow[t]{3}{*}{ Konsistenz } & Derb & 6,7 & 5,3 \\
\hline & Mittelfest & 0 & 26,3 \\
\hline & Weich & 93,3 & 68,4 \\
\hline
\end{tabular}

hypo- und hyperpigmentierte Narben ähnlich oft auftraten (27\% und 33\% Tab. 5). In beiden Gruppen war der Anteil von normal pigmentierten Narben mit 37 und 40\% annähernd gleich hoch (० Abb. 8).

Das Narbenrelief war in der Sekundärheilungsgruppe vorwiegend eingesunken (53\%), jedoch in der Limberglappengruppe vermehrt erhaben (37\%) ( $\bullet$ Abb. 8).

Die Konsistenz der Narben war in beiden Gruppen zum größten Teil weich (Sekundärheilung 93\%, Limberglappen 68\%), festere bis derbe Narben traten zusammengenommen mit 32\% in der Limberglappengruppe häufiger auf ( $\bullet$ Abb. 8).

\section{Diskussion}

$\nabla$

In der vorliegenden Studie ließ sich, unseres Wissens nach erstmals zeigen, wie sich eine Limberglappenplastik im Vergleich zur Sekundärheilung positiv auf die Narbenqualität nach Pilonidalsinusexzision auswirkt.

\section{Narbenqualität}

Wir konnten Vorteile der Limberglappenplastik hinsichtlich der Hautdehnbarkeit und Verschieblichkeit im Narbenbereich nachweisen, die im Nachfolgenden erörtert werden.

Die Dehnung der Strecke AB, welche sich in der Sinus pilonidalis Region befindet, wies zwischen allen 3 Gruppen signifikante Unterschiede auf, mit den niedrigsten Werten in der Sekundärheilungsgruppe, darauffolgend der Limberglappengruppe und den höchsten Werten in der gesunden Kontrollgruppe.

Die gesunde Referenzhaut im Bereich der Strecke CD dehnte sich in allen Gruppen ähnlich ( $\odot$ Tab. 4). Das bedeutet, dass die Ausgangselastizität der Haut aller Probanden gut vergleichbar war. Die Unterschiede der Hautdehnbarkeit in der Narbenregion sind somit auf die Behandlung zurückzuführen.

Diese These wird auch durch die Ergebnisse der relativen Dehnbarkeit (SLQ) bestätigt. Hierbei wurde jeweils im selben Individuum, durch die o.g. mathematische Formel, die Dehnbarkeit der Haut im Narbenbereich mit gesunder Haut kranial des Narbenbereichs verglichen. Der SLQ fiel in der Sekundärheilungsgruppe signifikant schlechter aus als in der Limberglappen- und Kontrollgruppe. Das bedeutet, dass sich die Haut im Narbenbereich nach der Sekundärheilung deutlich schlechter dehnte, als die gesunde Haut des jeweils selben Probanden, im Vergleich zur Limberg- und Kontrollgruppe. Nach der Limberglappenplastik hingegen, erreichte die Haut im Narbenbereich eine relative Dehnungsfähigkeit, die derjenigen in der gesunden Kontrollgruppe entsprach. 


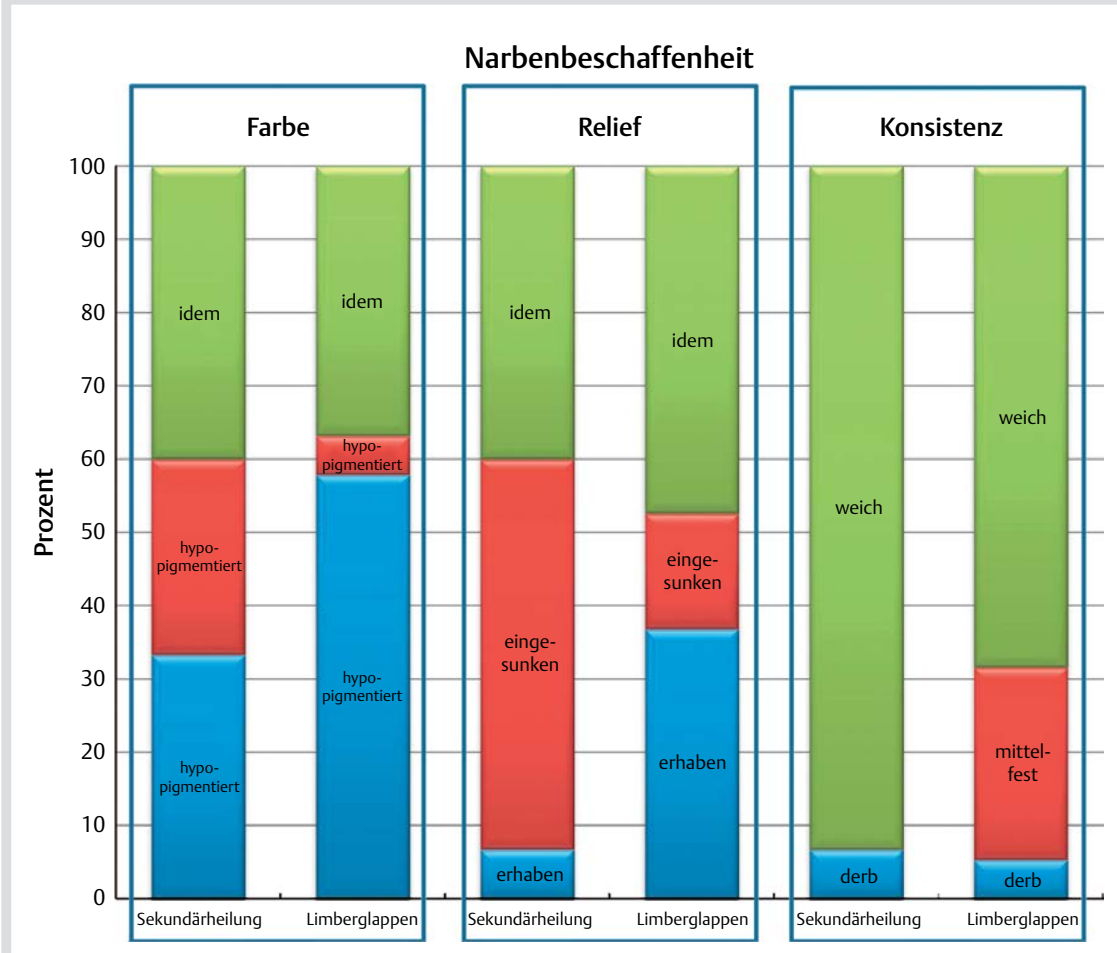

Abb. 8 Narbenbeschaffenheit

Als zweiten Parameter der Hautelastizität untersuchten wir die Verschieblichkeit der Haut im Narbenbereich. Hier konnte ebenfalls ein Vorteil der Limberglappenplastik nachgewiesen werden. Die Hautverschlieblichkeit in der Sekundärheilungsgruppe war signifikant schlechter als in der Kontrollgruppe, während sie in der Limberglappengruppe der gesunden Kontrollgruppe entsprach.

Uns sind keine anderen Studien bekannt, die sich mit der Narbenqualität nach der Behandlung des Sinus pilonidalis, oder anderen Eingriffen in der Sakralregion, beschäftigen. Somit handelt es sich hierbei unseres Wissens nach um eine Erstbeschreibung, die einen Vergleich mit der bisherigen Literatur nicht möglich macht.

\section{Schmerzen, Arbeitsausfall, Zufriedenheit}

Die Gruppen unterschieden sich bezüglich der Schmerzen nicht, sie wiesen dieselben Mittelwerte auf der Numerischen Analogskala auf. In beiden Patientengruppen bestanden im Durchschnitt keine Ruheschmerzen und nur sehr geringe Belastungsschmerzen. In einer Studie von Jamal et al. wurden sogar geringere postoperative Schmerzen nach Limberglappenplastik im Vergleich zur Sekundärheilung beschrieben [25].

Die Arbeitsausfallzeiten wichen hingegen deutlich voneinander ab. Auch wenn dieser Unterschied aufgrund einer hohen Standardabweichung statistisch keine Signifikanz erlangte, waren damit die Patienten der Sekundärheilungsgruppe effektiv mehr als doppelt so lange und damit ca. 1 Monat länger arbeitsunfähig, im Vergleich zur Limberglappengruppe. Dieser Unterschied wurde ebenfalls von Rashidian et al. [16] bestätigt. Ursächlich für die kürzere Arbeitsunfähigkeit ist sicher unter anderem die geringere Heilungsdauer nach der Limberglappenplastik $[16,33]$, wie sie auch in einer Meta-Analyse von Al-Khamis et al. [10] nachgewiesen wurde. Hierbei sollte erwähnt werden, dass einige Patienten der Sekundärheilungsgruppe in der Befragung angaben, mit noch nicht verschlossener Wunde bereits die Arbeit wiederaufgenommen zu haben.

In beiden Patientengruppen war die Zufriedenheit mit dem Behandlungsverlauf und dem ästhetischen Ergebnis hoch. Eine Tendenz zur höheren Zufriedenheit bestand sowohl für den Behandlungsverlauf als auch für das ästhetische Ergebnis in der Limberglappengruppe, ohne jedoch statistische Signifikanz zu erreichen. In einer Studie von Holmebakk et al. wurde ebenfalls kein signifikanter Unterschied in der Zufriedenheit mit dem ästhetischen Ergebnis und der Gesamtzufriedenheit festgestellt [26].

\section{Vergleichbarkeit der Gruppen}

Unsere Patientengruppen erwiesen sich bezüglich der epidemiologischen Voraussetzungen und des Rauchverhaltens als gut vergleichbar. Es bestanden zwar formal signifikante Unterschiede in Alter und Größe zwischen den Gruppen, jedoch waren diese aufgrund ihrer geringen Ausprägung klinisch nicht relevant. Der Altersunterschied betrug im Mittel 2 Jahre und der Größenunterschied $1 \mathrm{~cm}$.

Zur Beurteilung der Narben zogen wir eine Kontrollgruppe von 100 gesunden Probanden heran, welche keine Narben am Rücken oder Bewegungseinschränkungen aufwiesen. Die epidemiologischen Daten und die Verteilung des BMI im Probandenkollektiv entsprachen annähernd denen in der deutschen Bevölkerung [34], wir können somit von einer repräsentativen Stichprobe der regionalen Bevölkerung ausgehen. Gegenüber der gesunden Kontrollgruppe wiesen beide Patientengruppen einen höheren BMI auf. Dies lässt sich durch die Adipositas als Pilonidalsinus-Risikofaktor erklären. Ebenso zu verstehen ist, dass die Patienten in der Limberglappengruppe signifikant jünger waren als die Probanden der Kontrollgruppe, da der Altersgipfel des Pilonidalsinus in der Pubertät bzw. Adoleszenz liegt [7]. Der Altersunterschied erlangte zwischen Sekundärheilungsgruppe und Kontrollgruppe keine Signifikanz. 


\section{Studiendesign}

Bei unserer Studie handelt es sich um eine prospektive Nachuntersuchung. Eine Randomisierung war nicht vorgesehen, da wir den Patienten die Möglichkeit geben wollten, nach ausführlicher Aufklärung selbst zu entscheiden, welche Behandlungsform durchgeführt wurde.

Die hier verglichenen Therapieverfahren wurden ausgewählt, da sie in unserem Hause parallel und mit jeweils konstant guten Ergebnissen praktiziert werden. Zudem gibt es bislang keine einheitliche Therapiempfehlung [1]. In der Literatur finden sich widersprüchliche Studienergebnisse im Vergleich der Therapieverfahren. Metaanalysen $[11,35]$ zufolge sind paramediane Verfahren wie Limberg- oder Karydakislappenplastik der Mittelliniennaht bezüglich Infektionen und Dehiszenzen überlegen. Aufgrund der bekannten, schlechten Studienergebnisse führen wir keine Mittelliniennähte durch. Als off-midline Verfahren kam bei uns der Limberglappen zum Einsatz. Einigen neueren Studien zufolge verspricht eine Modifikation der Limberglappenplastik durch exzentrischen Versatz des unteren Narbenpols sogar noch bessere Ergebnisse bezüglich Rezidiven [36], Wundheilungsstörungen und Infektionen [37].

\section{Fazit}

$\nabla$

Die Limberglappenplastik eignet sich gut zur Defektdeckung nach Pilonidalsinusexzision. Sie ist der Sekundärheilung bezüglich der Hautelastizität im Narbenbereich und der Arbeitsunfähigkeitsdauer überlegen. Eine Modifikation der Limberglappenplastik durch Lateralisation des unteren Wundpols führen wir nun bereits durch und werden die weiteren Ergebnisse beobachten. Da Patientenzufriedenheit und Schmerzen nach Sekundärheilung vergleichbar mit denen nach Limberglappenplastik sind, überlassen wir nach ausführlicher Aufklärung weiterhin den Patienten die Wahl des Vorgehens.

Durch die Untersuchung eines größeren Patientenkollektivs und einen längeren Nachuntersuchungszeitraum könnten zukünftig möglicherweise noch mehr signifikante Unterschiede aufgezeigt werden. Dies wären z. B. positive Meßwerte in Bezug auf die Narbenqualität und klinische Parameter wie Zufriedenheit und Schmerzen nach Limberglappenplastik.

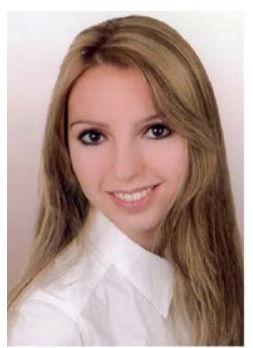

\section{Kurzbiographie}

Sonja Dahmann

In Mainz geboren. Medizinstudium von 2004-2010 und Promotion 2011 an der Charité - Universitätsmedizin Berlin. Seit 2011 Assistenzärztin in der Klinik für Plastische, Hand- und Wiederherstellungschirurgie unter der Leitung von Chefarzt PD Dr. Meyer-Marcotty im KlinikumStadtSoest.

\section{Literatur}

1 Arbeitsgemeinschaft der Wissenschaftlichen Medizinischen Fachgesellschaften. S3-Leitlinie: Sinus pilonidalis. 04/2014 (zitiert am 12.01.2015). URL http://www.awmf.org/leitlinien/detail/1l/081-009. html

2 Bascom J. Pilonidal disease: origin from follicles of hairs and results of follicle removal as treatment. Surgery 1980; 87: 567-572

3 Bolandparvaz S, Moghadam Dizaj P, Salahi $R$ et al. Evaluation of the risk factors of pilonidal sinus: a single center experience. Turk J Gastroenterol 2012; 23: 535-537

4 Stelzner F. Die Ursache des Pilonidalsinus und der Pyodermia fistulans sinifica. Langenbecks Arch Chir 1984; 362: 105-118

5 Søndenaa K, Andersen E, Nesvik I et al. Patient characteristics and symptoms in chronic pilonidal sinus disease. International Journal of Colorectal Disease 1995; 10: 39-42

6 Wang C, Yao Y, Cao Y. The integrative method "suture dragging and simplified vacuum assisted therapy" for complex pilonidal sinus disease. Case Rep Surg 2014, 2014:425497 doi:10.1155/2014/425497 Epub 2014 Mar 4

7 Doll D, Friederichs J, Dettmann $H$ et al. Time and rate of sinus formation in pilonidal sinus disease. Int J Colorectal Dis 2008; 23: 359-364

8 Sondenaa K, Andersen E, Nesvik I et al. Patient characteristics and symptoms in chronic pilonidal sinus disease. Int J Colorectal Dis 1995; 10: 39-42

9 Aysan E, Ilhan M, Bektas $H$ et al. Prevalence of sacrococcygeal pilonidal sinus as a silent disease. Surg Today 2013; 43: 1286-1289 doi:10.1007/s00595-012-0433-0 Epub 2012 Nov 25

10 Al-Khamis A, McCallum I, King PM et al. Healing by primary versus secondary intention after surgical treatment for pilonidal sinus. Cochrane Database Syst Rev 2010; 20: CD006213 doi:10.1002/14651858. CD006213.pub3

11 Enriquez-Navascues JM, Emparanza JI, Alkorta $M$ et al. Meta-analysis of randomized controlled trials comparing different techniques with primary closure for chronic pilonidal sinus. Tech Coloproctol 2014; 18 : 863-872 doi:10.1007/s10151-014-1149-5 Epub 2014 Apr 30

12 Maurice BA, Greenwood RK. A Conservative Treatment of Pilonidal Sinus. Br J Surg 1964; 51: 510-512

13 Colov EP, Bertelsen CA. Short convalescence and minimal pain after out-patient Bascom's pit-pick operation. Dan Med Bull 2011; 58: A4348

14 Soll C, Dindo D, Steinemann $D$ et al. Sinusectomy for primary pilonidal sinus: less is more. Surgery 2012; 150: 996-1001

15 Blanco $G$, Giordano $M$, Torelli I. Surgical treatment of pilonidal sinus with open surgical technique. Minerva Chir 2003; 58: 181-187

16 Rashidian N, Vahedian-Ardakani J, Baghai-Wadji $M$ et al. How to repair the surgical defect after excision of sacrococcygeal pilonidal sinus: a dilemma. J Wound Care 2014; 23: 630-633 doi:10.12968/ jowc.2014.23.12.630

17 Gwynn BR. Use of the rhomboid flap in pilonidal sinus. Ann R Coll Surg Engl 1986; 68: 40-41

18 Kanat BH, Bozan MB, Yazar FM et al. Comparison of early surgery (unroofing-curettage) and elective surgery (Karydakis flap technique) in pilonidal sinus abscess cases. Ulus Travma Acil Cerrahi Derg 2014; 20: 366-370 doi:10.5505/tjtes.2014.62547

19 Karydakis GE. New approach to the problem of pilonidal sinus. Lancet 1973; 2: 1414-1415

20 Bascom JU. Repeat pilonidal operations. Am J Surg 1987; 154: 118-122

21 Fazeli MS, Adel MG , Lebaschi AH et al. Comparison of outcomes in Z-plasty and delayed healing by secondary intention of the wound after excision of the sacral pilonidal sinus: results of a randomized, clinical trial. Dis Colon Rectum 2006; 49: 1831-1836

22 Morrison PD. Is Z-plasty closure reasonable in pilonidal disease? Ir J Med Sci 1985; 154: 110-112

23 Yazar M, Kurt Yazar S, Celet Ozden B et al. Cosmetic closure of pilonidal sinus defects with bilateral transpositionale adipofascial flaps. J Plast Surg Hand Surg 2013; 4 [Epub ahead of pint]

24 Unalp HR, Derici H, Kamer E et al. Lower recurrence rate for Limberg vs. V-flap for pilonidal sinus. Dis Colon Rectum 2007; 50: 1436-1444

25 Jamal A, Shamim M, Hashmi $F$ et al. Open excision with secondary healing versus rhomboid excision with Limberg transposition flap in the management of sacrococcygeal pilonidal disease. J Pak Med Assoc 2009; 59: 157-160

26 Holmebakk T, Nesbakken A. Sugery for pilonidal disease. Scandinavian Journal of Surgery 2005; 94: 43-46

27 Winn HR, Jane JA, Rodeheaver $G$ et al. Influence of subcuticular sutures on scar formation. Am J Surg 1977; 133: 257-259 
28 Berry DP, Harding KG, Stanton MR et al. Human wound contraction: collagen organization, fibroblasts, and myofibroblasts. Plast Reconstr Surg 1998; 102: 124-131 discussion 132-134

29 Dematte MF, Gemperli $R$, Salles AG et al. Mechanical evaluation of the resistance and elastance of post-burn scars after topical treatment with tretinoin. Clinics 2011; 66: 1949-1954

30 Firooz A, Sadr B, Babakoohi S et al. Variation of Biophysical Parameters of the Skin with Age, Gender, and Body Region. The Scientific World Journal Volume 2012 2012: 386936

31 Nerlich M, Berger A., (Hrsg.). Tscherne Unfallchirurgie. Weichteilverletzungen und -infektionen. Berlin Heidelberg: Springer; 2003: 4-5

32 Dahmann S, Lebo PB, Görlich D et al. Sakrale Hautelastizität - Etablierung einer noninvasiven Messmethode. DOI: 10.1055/s-0042-104118

33 Karakayali F, Karagulle E, Karabulut $Z$ et al. Unroofing and marsupialization vs. rhomboid excision and Limberg flap in pilonidal disease: a prospective, randomized, clinical trial. Dis Colon Rectum 2008; 52: 496-502
34 Statistisches Bundesamt (Destatis). Gesundheitsrelevantes Verhalten. Körpermaße nach Altersgruppen 2009;(02.06.2010). Im Internet https://www.destatis.de/DE/ZahlenFakten/GesellschaftStaat/Gesund heit/GesundheitszustandRelevantesVerhalten/Tabellen/Koerpermas se.html Stand 06.02.2014

35 Horwood J, Hanratty D, Chandran P et al. Primary closure or rhomboid excision and Limberg flap for the management of primary sacrococcygeal pilonidal disease? A meta-analysis of randomized controlled trials. Colorectal Dis 2012; 14: 143-151

36 Mentes BB, Leventoglu S, Cihan A et al. Modified Limberg transposition flap for sacrococcygeal pilonidal sinus. Surg Today 2004; 34: 419-423

37 Cihan A, Ucan BH, Comert M et al. Superiority of asymmetric modified Limberg flap for surgical treatment of pilonidal disease. Dis Colon Rectum 2006; 49: 244-249 